\title{
Influence of Maternal Environmental Tobacco Smoke Exposure Assessed by Hair Nicotine Levels on Birth Weight
}

\author{
Jungun Lee ${ }^{1,2}$, Dong-Ryul Lee ${ }^{1}$, Do-Hoon Lee ${ }^{3}$, Yu-Jin Paek ${ }^{4 *}$, Won-Chul Lee ${ }^{2 *}$
}

\begin{abstract}
Background: While the perinatal outcomes of active maternal smoking are well documented, results of the effects of environmental tobacco smoke (ETS) exposure during pregnancy are inconsistent. We aimed to examine the effect of ETS exposure, assessed by maternal hair nicotine levels at $35^{\text {th }}$ week of gestation, on birth weight and the risk of small for gestational age (SGA) and low birth weight (LBW). Materials and Methods: A total of 871 non-smoking healthy pregnant women were recruited by one Korean hospital between 1 October 2006 and 31 July 2007. Hair samples were collected and anthropometric questionnaires administered at $35^{\text {th }}$ week of gestation. The primary outcome was birth weight and secondary outcomes were the risk of babies being SGA and LBW. Results: Log-transformed hair nicotine concentrations were inversely related with birth weight after adjusting for confounding variables $(\beta=-0.077, p=0.037)$. After stratifying hair nicotine levels by tertiles $(\mathrm{T} 1$, low [0.0-0.28 ng/mg]; T2, medium [0.29-0.62 ng/mg]; and T3, high [0.63-5.99 ng/mg]), the mean birth weight in each groups were 3,342g (T1) 3,296g (T2) and 3,290 g (T3), respectively. However the difference between groups was not statistically significant by analysis of co-variance (ANCOVA) adjusting for covariates $(\mathrm{p}=\mathbf{0 . 0 6 2})$. In logistic regression analysis, the risk of SGA was higher in the T3 (OR=1.59, 95\% CI 1.05-2.42) than in the reference group (T1), after controlling for confounding variables. The risk of low birth weight $(<2,500 \mathrm{~g}, \mathrm{LBW})$ was not significantly higher $(\mathrm{OR}=1.44,95 \% \mathrm{CI} 0.95-2.19)$, but the risk of babies being below 3,000g birth weight was increased in the $\mathrm{T} 3$ group $(\mathrm{OR}=1.53,95 \% \mathrm{CI}$ 1.00-2.36) compared with that in the T1 group. Conclusions: Maternal ETS exposure during pregnancy was inversely related with birth weight. The risk of SGA increased in the highest ETS exposure group compared with in the low exposure group. To prevent ETS exposure during pregnancy, more comprehensive tobacco control policies are needed.
\end{abstract}

Keywords: Birth weight - hair nicotine - small for gestational age - environmental tobacco smoke - second hand smoke

Asian Pac J Cancer Prev, 16 (7), 3029-3034

\section{Introduction}

Active maternal smoking has well-characterized adverse effects on several outcomes, including infertility, sudden infant death syndrome (SIDS), and child growth and development (Surgeon General's Report, 2006). However, the effects of passive maternal smoking, also known as environmental tobacco smoke (ETS) exposure, have been less well studied, with several studies showing inconsistent results (Salmasi et al., 2010). In addition, there is some evidence indicative of associations of childhood cancer with secondhand smoke exposure although the evidence is inadequate for some sources and periods of exposure (Surgeon General's Report, 2006).

Recently, an association between passive maternal smoking during pregnancy and low birth weight (LBW) has been suggested, as birth weight has been found to be $25-75 \mathrm{~g}$ lower in the off-spring of mothers with ETS exposure (Misra and Nguyen, 1999; Leonardi-Bee et al., 2008; Salmasi et al., 2010).

Since the enactment of the National Health Promotion Act in Korea in 1995, the smoking rate in Korean men has shown a 4-5\% annual reduction up to 2009 (Kim et al., 2013; Park et al., 2013; Kim et al., 2014). Despite this reduction, smoking rate is still quite high among men in Korea compared with that from other OECD countries. In a national survey conducted in 2008 , the smoking rate in women was $6.6-7.4 \%$ by self-reporting, but increased to $11.4-14.5 \%$ assessed by urine cotinine which means that smoking rates of women in Korea are higher than previously thought (Kang et al., 2013; Lee et al., 2014).

Despite having one of the highest smoking rates among men worldwide, information on second hand smoke (SHS) exposure on adults and children is lacking in Korea (Hughes et al., 2008b). One population-based telephone survey conducted in 332 non-smoking adults

${ }^{1}$ Department of Family Medicine, Sanbon Medical Center, Wonkwang University, College of Medicine, ${ }^{3}$ Center for Clinical Services, National Cancer Center, Goyang-si, ${ }^{4}$ Department of Family Medicine and Health Promotion Center, Sacred Heart Hospital, Hallym University, College of Medicine, Anyang, Gyeonggi-do, ${ }^{2}$ Department of Public Health, The Catholic University of Korea, Graduate School, Seoul, Republic of Korea*For correspondence:paek@hallym.or.kr,leewc@catholic.ac.kr 
in Seoul revealed that $65 \%$ of non-smokers were exposed to SHS during a typical day. Exposure was most common in locations other than home compared with work and home (57\% vs $26 \%$ and $25 \%$ of respondents) (Hughes et al., 2008a).

As of 2004, $40 \%$ of children, $33 \%$ of non-smoking men, and $35 \%$ of non-smoking women worldwide were exposed to ETS (Oberg et al., 2011; Park et al., 2013). In Taiwan, $58 \%$ of pregnant women and $57 \%$ of those who had never smoked during their reproductive age were shown to have been exposed to ETS either at work or at home (Wen et al., 2005).

The precise evaluation of cigarette consumption of women smokers in epidemiological studies is difficult and may explain discrepancies between studies. Details on cigarette consumption have often been collected only once at the first prenatal visit or consumption has been classified as a dichotomous variable (Jacqz-Aigrain et al., 2002).

Nicotine and its major metabolite, cotinine, are commonly used as smoking biomarkers, and their levels can be determined in various biological specimens such as blood, saliva, and urine (Seong et al., 2007). Recently, hair analysis was found to be a convenient, noninvasive technique for detecting nicotine exposure. Because nicotine/cotinine accumulates in hair during hair growth, it is a unique measure of long-term, cumulative exposure to tobacco smoke (Hwang et al., 2012).

In this study, we aimed to examine the association between ETS, assessed by maternal hair nicotine levels at $35^{\text {th }}$ week of gestation, and birth weight. In addition, we investigated the association between ETS and the risk of babies being small for gestational age (SGA) or LBW.

\section{Materials and Methods}

\section{Study population}

All pregnant women receiving prenatal care between 1 October 2006 and 31 July 2007 at the ambulatory antenatal clinic of an obstetrics and gynecology specialty hospital in an urban area were invited to participate in this study. The study was designed to determine the effect of ETS in nonsmoking pregnant women whose spouses smoke outside the home as described previously (Yoo et al., 2010). The inclusion criteria were healthy pregnant women at $35^{\text {th }}$ week of gestation with singleton pregnancies who reported no history of smoking or who quit smoking before

Table 1. Baseline Characteristics of Mothers and their Newborns Stratified by Maternal Hair Nicotine Concentration Tertiles

\begin{tabular}{|c|c|c|c|c|}
\hline \multirow{2}{*}{ Variables } & \multicolumn{3}{|c|}{ Hair nicotine concentration (ng/mg) } & \multirow{3}{*}{$\mathrm{p}$} \\
\hline & $\begin{array}{l}\text { Tertile } 1 \\
(n=300)\end{array}$ & $\begin{array}{l}\text { Tertile } 2 \\
(n=289)\end{array}$ & $\begin{array}{l}\text { Tertile } 3 \\
(n=282)\end{array}$ & \\
\hline Hair nicotine concentration, [ng/mg] & {$[0.0-0.28]$} & {$[0.29-0.62]$} & {$[0.63-5.99]$} & \\
\hline Maternal age, years & & & & 0.13 \\
\hline $20-29$ & $115(38.4)$ & $93(31.6)$ & $108(38.9)$ & \\
\hline$\geq 30$ & $185(61.6)$ & $201(68.4)$ & $169(61.1)$ & \\
\hline Maternal body weight before pregnancy, $\mathrm{kg}^{*}$ & $54.9 \pm 7.04$ & $56.4 \pm 7.87$ & $57.6 \pm 8.45$ & $<0.001$ \\
\hline Maternal BMI before pregnancy, $\mathrm{kg} / \mathrm{m} 2 *$ & $21.3 \pm 2.60$ & $21.8 \pm 2.81$ & $22.2 \pm 3.08$ & $<0.001$ \\
\hline Maternal weight at delivery, $\mathrm{kg}^{*}$ & $65.3 \pm 7.64$ & $66.6 \pm 7.69$ & $68.4 \pm 8.28$ & $<0.001$ \\
\hline Occupation & & & & 0.18 \\
\hline Housewives & $84(27.9)$ & $74(25.2)$ & $58(21.1)$ & \\
\hline Other & $218(72.1)$ & $219(74.8)$ & $217(78.9)$ & \\
\hline Recent alcohol consumption** & & & & 0.007 \\
\hline Yes & $11(3.5)$ & 14 (4.8) & $27(9.5)$ & \\
\hline No & $287(96.5)$ & $272(95.2)$ & $261(90.5)$ & \\
\hline Education & & & & $<0.001$ \\
\hline High school or lower & $51(16.9)$ & $56(19.3)$ & $96(33.8)$ & \\
\hline College or higher & $249(83.1)$ & $232(80.7)$ & $188(66.2)$ & \\
\hline Monthly income, Korean Won & & & & $<0.001$ \\
\hline$<2$ million & $51(16.9)$ & $56(19.3)$ & $96(33.8)$ & \\
\hline 2-4 million & $224(75.0)$ & $213(74.0)$ & $171(60.1)$ & \\
\hline$>4$ million & $24(8.1)$ & $19(6.7)$ & $17(6.1)$ & \\
\hline Delivery type & & & & 0.004 \\
\hline NSD & $220(73.3)$ & $198(68.8)$ & $172(60.6)$ & \\
\hline CS & $80(26.7)$ & $90(31.2)$ & $111(39.4)$ & \\
\hline Neonatal sex & & & & 0.17 \\
\hline Male & $148(49.3)$ & $156(54.0)$ & $130(46.1)$ & \\
\hline Female & $152(50.7)$ & $133(46.0)$ & $152(53.9)$ & \\
\hline Gestational age, weeks* & & & & 0.037 \\
\hline & $39.7 \pm 1.01$ & $39.5 \pm 1.10$ & $39.5 \pm 1.06$ & \\
\hline Parity & & & & 0.39 \\
\hline 0 & $162(54.0)$ & $170(58.8)$ & $165(58.5)$ & \\
\hline 1 & $130(43.3)$ & 109 (37.7) & $104(36.9)$ & \\
\hline$\geq 2$ & $8(2.7)$ & $10 \quad(3.5)$ & $13(4.6)$ & \\
\hline
\end{tabular}

*Data were calculated by analysis of variance (ANOVA); other variables were analyzed by chi-square test; **Drinking alcohol within 1 month. Continuous variables are shown as mean \pm S.D (Standard deviation) or [range] and categorical variables as numbers (percentage). NSD: normal spontaneous delivery; CS: cesarean section 
pregnancy, visited the aforementioned clinic at least twice for antenatal care, and had available birth outcome details, including birth weight.

A total 993 women agreed to participate in the study. Participants with unavailable birth weight $(n=65)$ and pregnancy-induced hypertension $(n=4)$, gestational diabetes $(\mathrm{n}=11)$, hyperthyroidism $(\mathrm{n}=1)$, hypothyroidism $(n=2)$, abnormal thyroid-stimulating hormone level $(n=32)$, and glomerular disease $(n=1)$ were excluded. Women with hair nicotine levels exceeding the predetermined level for smokers (6.40 ng/mg hair) in the previous study were also excluded (Yoo et al., 2010). The final study sample included in the analysis consisted of 871 women.

This study was approved by the Institutional Review Board of the Hallym University Sacred Heart Hospital (Hallym Research No. 811e2010), and each participant provided written consent to participate in the study.

\section{Questionnaire information}

Information about maternal age, educational level, monthly income, alcohol consumption per month, occupation, and parity (zero, one, or more) was obtained by administering a questionnaire at study enrollment. Maternal height and weight before pregnancy were measured without shoes or heavy clothing.

Neonatal morphometric birth weight measurement was performed immediately after delivery. Information about neonatal sex, delivery type, and maternal weight at delivery was collected from hospital medical records.

The main outcomes were birth weight in grams, SGA, and LBW $(<2,500 \mathrm{~g})$. SGA was defined as birth weight in the lowest 10th percentile according to the week of

Table 2. Association between Maternal Hair Nicotine Levels and Birth Weight Analyzed by Multiple Linear Regression

\begin{tabular}{lcccc}
\hline & \multicolumn{4}{c}{ Birth weight } \\
& $\beta$ & $\mathrm{p}$ & $\beta$ & $\mathrm{p}$ \\
\hline Hair nicotine concentration & -0.078 & 0.026 & -0.077 & 0.037 \\
Maternal BMI & 0.17 & $<0.001$ & 0.17 & $<0.001$ \\
Neonatal sex & -0.1 & 0.003 & -0.1 & 0.005 \\
Gestational age & 0.38 & $<0.001$ & 0.38 & $<0.001$ \\
Maternal age & -0.62 & 0.53 & -0.024 & 0.51 \\
Delivery type & -0.024 & 0.49 & -0.018 & 0.62 \\
Parity & & & 0.006 & 0.53 \\
Education & & & 0.16 & 0.031 \\
Income & & & -0.14 & 0.062 \\
Occupation & & & -0.014 & 0.7 \\
\hline
\end{tabular}

*All regression coefficients $(\beta)$ were statistically significant at $\mathrm{p}<0.05$; BMI: body mass index

Table 3. Association between Birth Weight and Maternal Hair Nicotine Concentration

\begin{tabular}{|c|c|c|c|c|}
\hline & \multicolumn{3}{|c|}{ Hair nicotine concentration, [ng/mg] } & \multirow[b]{2}{*}{$\mathrm{p}$} \\
\hline & $\begin{array}{c}\text { Tertile } 1 \\
{[0.0-0.28]}\end{array}$ & $\begin{array}{c}\text { Tertile } 2 \\
{[0.29-0.62]}\end{array}$ & $\begin{array}{c}\text { Tertile } 3 \\
{[0.63-5.99]}\end{array}$ & \\
\hline Birth weight, $g$ & $3.342 \pm 24$ & $3.296 \pm 25$ & $3.290 \pm 25$ & 0.062 \\
\hline \multicolumn{5}{|c|}{$\begin{array}{l}\text { *Data were calculated by analysis of covariance (ANCOVA) adjusted } \\
\text { for maternal age, maternal BMI, fetal sex, gestational age, delivery type, } \\
\text { parity, education, income, and occupation. Values are given as mean } \pm \text { SD } \\
\text { (Standard deviation) or [range] }\end{array}$} \\
\hline
\end{tabular}

gestation. We calculated sex-and gestational age-specific (SGA) z scores from birth weight and gestation data using the current Korean population source data of non-smoking women (Lee, 2007). Since this weight measure is adjusted for gestational age at birth, it can also be considered a measure of fetal growth in utero. LBW was categorized as LBW1 or LBW2 if birth weights were $<2,500 \mathrm{~g}$ or $3,000 \mathrm{~g}$, respectively.

\section{Hair nicotine analysis}

Hair nicotine levels were analyzed by liquid chromatography-tandem mass spectrometry (LC-MS/ MS), as described previously (Ryu et al., 2006). Briefly, $1 \mathrm{mg}$ hair sample was finely cut and digested with 0.90 $\mathrm{mL}$ of a $1 \mathrm{~mol} / \mathrm{L} \mathrm{NaOH}$ solution. Digested samples were mixed with internal standards and extracted with $2 \mathrm{~mL}$ of diethyl ether. Extracts were evaporated for $40 \mathrm{~min}$ at $50^{\circ} \mathrm{C}$, re-dissolved in $50 \mathrm{~mL}$ of a methanol/water mobile phase $(80: 20, \mathrm{v} / \mathrm{v})$, and injected $(10 \mathrm{~mL})$ into the LC-MS/ MS system. The limit of detection for hair nicotine level was $0.16 \mathrm{ng} / \mathrm{mg}$ hair for all analytes (Yoo et al., 2010).

\section{Statistical analysis}

As the distribution of hair nicotine levels was positively skewed, a common log transformation was applied to the data. The results of descriptive statistics regarding hair nicotine levels are presented as geometric means and 95\% confidence intervals (CI) (Yoo et al., 2010). Maternal hair nicotine concentrations were stratified by tertiles as low (T1;0.0-0.28 ng/mg), medium (T2; 0.29-0.62 ng/mg), and high (T3; 0.63-5.99 ng/mg). The characteristics of the mother and baby were analyzed according to nicotine tertiles using chi-square tests and analysis of variance. Multiple linear regression analysis was performed to investigate the change of birth weight according to log-transformed hair nicotine concentrations after adjusting for confounding variables. Comparison of mean birth weight according to hair nicotine concentration categories was carried out by analysis of co-variance (ANCOVA), adjusting for confounding variables. Finally, multiple logistic regression was used to evaluate the effect of different nicotine exposure levels, defined by hair nicotine concentration categories, on the dichotomous outcomes (SGA, LBW1, LBW2).

All statistical analyses were performed using the SPSS V.16.0 software (SPSS, Chicago, Illinois, USA). For all comparisons, two-tailed $\mathrm{p}$ values $<0.05$ were considered significant, and CIs were calculated at the $95 \%$ level.

\section{Results}

Baseline study population characteristics of the T1, T2, and T3 groups are shown in Table 1. Maternal age, proportion of homemakers, neonatal sex, and maternal parity were similar among the three groups.

Maternal body weight and body mass index (BMI) before pregnancy and at delivery were higher in the T3 group than in the other groups, but the gestational age was higher in the T1 group. Recent alcohol consumption during pregnancy was more common in the T3 group, but a higher level of education, income, and normal delivery 
Table 4. Adjusted OR of Small for Gestation age (SGA) and Low Birth Weight (LBW) According to the Tertile of the Maternal Hair Nicotine Concentration by Multiple Logistic Regression

\begin{tabular}{lllclcrr}
\hline & & \multicolumn{2}{c}{ SGA } & \multicolumn{2}{c}{ LBW1** } & \multicolumn{2}{c}{ LBW2*** } \\
& & OR & 95\% CI & OR & $95 \%$ CI & OR & $95 \%$ CI \\
\hline Nicotine Tertile & Tertile 1 & 1 & & 1 & & 1 & \\
& Tertile 2 & 1.21 & $0.79-1.84$ & 1.03 & $0.67-1.56$ & 1.01 & $0.65-1.57$ \\
& Tertile 3 & 1.59 & $1.05-2.42$ & 1.44 & $0.95-2.19$ & 1.53 & $1.00-2.36$ \\
\hline
\end{tabular}

*SGA: small for gestational age; **LBW1: low birth weight ( $<2,500 \mathrm{~g})$; ***LBW2: low birth weight ( $<3,000 \mathrm{~g})$; OR: odds ratio; CI: confidence interval; Adjusted variables: maternal age, maternal BMI, fetal sex, gestational age, delivery type, parity, education, income, and occupation

rate was more frequent in the $\mathrm{T} 1$ group (Table 1).

Log-transformed hair nicotine concentrations were significantly and inversely correlated with birth weight after adjusting for maternal BMI, neonatal sex, gestational and maternal age, and delivery type (Model 1, $\beta=-0.078$, $\mathrm{p}=0.026$ ), and after controlling for additional variables such as parity, education level, income, and occupation (Model 2, $\beta=-0.077, \mathrm{p}=0.037$ ) (Table 2).

Mean birth weight in each groups were $3,342 \mathrm{~g}$ (T1) 3,296 $\mathrm{g}$ (T2) and 3,290 $\mathrm{g}$ (T3), respectively, but differences among exposure groups were not statistically significant after adjusting for covariates using ANCOVA ( $\mathrm{p}=0.062)$ (Table 3).

In logistic regression analyses, the risk of having an SGA baby was higher in the T3 $(\mathrm{OR}=1.59,95 \%$ CI 1.05 2.42) than in the reference group (T1) (Table 4), after controlling for confounding factors. The risk of low birth weight $(<2,500 \mathrm{~g}, \mathrm{LBW} 1)$ was not significantly higher (OR=1.44, 95\%CI 0.95-2.19), but the risk of having birth weight below $3,000 \mathrm{~g}$ was increased in the T3 group $(\mathrm{OR}=1.53,95 \% \mathrm{CI} 1.00-2.36)$ compared with that in the T1 group.

\section{Discussion}

A decrease of fetal body weight was observed in proportion to the increase of log transformed maternal hair nicotine concentration. Mean fetal body weight was tend to be small in the high exposure group than the low exposure group, but it was marginally significant $(p=0.062)$. The risk of having an SGA baby, a reflection of intrauterine growth retardation (IUGR), was increased by $59 \%$ in subjects with high nicotine exposure. While the risk of low body weight $(<2,500 \mathrm{~g})$ did not significantly increase, but the risk of having a baby with birth weight less than $3,000 \mathrm{~g}$ was increased by $53 \%$ in the T3 group.

Results from previous studies including meta-analyses using dichotomous self-reporting questionnaires or cotinine as an exposure variable have suggested that the mean birth weight is 24-75g lower in exposed mothers (Lindbohm et al., 2002; Jaddoe et al., 2008; Leonardi-Bee et al., 2008). Contrary to previous studies, we showed a significant inverse dose-response relationship between ETS and birth weight and SGA using maternal hair nicotine concentrations as a measurement of nicotine exposure, as a continuous or categorical variable.

Some investigators have evaluated dose-response effects using hair nicotine levels. In a case-control study conducted in Norway (Nafstad et al., 1998), offspring of mothers with hair nicotine concentrations between the $25^{\text {th }}$ and $75^{\text {th }}$ percentiles (OR=3.2, 95\%CI 1.3-8.0), or above the $75^{\text {th }}$ percentile (OR=4.2, 95\%CI 1.5-11.5), had an increased risk of SGA relative to those with nicotine concentrations below the $25^{\text {th }}$ percentile. Furthermore, a 1.4 odds ratio increase (95\%CI 1.1-1.9) per unit of log-transformed maternal hair nicotine concentration was observed. However, another study (Jaakkola et al., 2001) failed to show an increased risk of having an LBW $(\mathrm{OR}=1.06,95 \% \mathrm{CI}$ 0.96-1.17) or SGA baby $(\mathrm{OR}=1.04$, 95\% CI 0.92-1.19) with high nicotine exposure group. But they used the cut-off values of hair nicotine concentration from that of the Nafstads. And the other studies that evaluated dose-response relationships did not find any trends (Rebagliato et al., 1995).

The results of our study provide evidence of a doseresponse relationship between ETS exposure and birth weight indicating that the effects of ETS might share the same biological mechanisms of active maternal smoking.

Previous studies have suggested that limitation of fetal growth by active maternal smoking results from reduced oxygen to the fetus, which is directly attributable to carbon monoxide exposure and nicotine-induced vasoconstriction, leading to reduced uterine and umbilical blood flow (Andres and Day, 2000; Surgeon General's Report, 2004). Some studies have shown that indicators of fetal hypoxia, such as nucleated red blood cell counts and erythropoietin levels, increased among women who smoked (Andres and Day, 2000) or were exposed to ETS during pregnancy (Dollberg et al., 2000).

Our findings showed that ETS exposure significantly increased the risk of SGA but not the risk of LBW, suggesting that the physiological effects of ETS are weaker than those of active maternal smoking. When we analyzed the risk of $<3,000 \mathrm{~g}$ birth weight rather than $<2,500 \mathrm{~g}$ of low birth weight (LBW) definition, the risk was increased $53 \%$. This result suggests that ETS impairs the fetal growth but the extent of the effect is attenuated than the active smoking that could cause LBW. SGA is caused by reduced fetal physical growth gestation that is either preterm or full term ( $\geq 37$ weeks of gestation), and may or may not be LBW.

Contrary to our results, a recent systematic review of the effects of ETS exposure on perinatal outcomes did not find a significant increase in SGA birth rates in both prospective and retrospective studies (OR 1.06, 95\%CI 0.75-1.50) (Salmasi et al., 2010). Moreover, results from two other meta-analyses did not show any association between ETS and SGA births (Lindbohm et al., 2002; Leonardi-Bee et al., 2008).

The established link between active maternal smoking and LBW is known to occur mainly through IUGR rather than through premature birth (Surgeon General's Report, 
2006). However, findings on the association between ETS and LBW appear to be inconsistent. A significant increase in LBW rate was found in 9 prospective studies (OR 1.32, 95\%CI 1.07-1.63) and 17 retrospective studies (OR 1.22, 95\%CI 1.08-1.37) (Leonardi-Bee et al., 2008). However, no association was found in a review of 19 additional studies (OR 1.16, 95\%CI 0.93-1.45) (Salmasi et al., 2010). These discrepancies might have originated from differences in controlling for important confounding variables such as maternal age, parity, socioeconomic status, alcohol, and drug use, as well as variability in the extent of adjustment between studies (Salmasi et al., 2010).

In our study the rate of low birth weight $(<2,500 \mathrm{~g})$ was low, and due to the high percentage of full term births and the low percentage of preterm births, we could not show an association between LBW and ETS. However, when 3,000 g was considered the cut-off of low birth weight, LBW was found to be associated with ETS.

The strength of our study lies in the use of hair nicotine concentration as a measurement of exposure to ETS, a more accurate marker reflecting long-term ETS exposure compared to cotinine or nicotine in serum, urine, or saliva samples. Thus, using this technique, we minimized errors due to recall bias, commonly seen with the use of selfadministered questionnaires in retrospective studies. In addition, the use of medical records and questionnaire information allowed us to take into account an extensive number of potential confounders.

Our study has several limitations. First, hair nicotine concentrations can be influenced by several factors such as air pollution and hair treatments, i.e., bleaching and dyeing. However, most pregnant women in Korea would hesitate to use hair treatments during pregnancy owing to concerns about harm to the fetus. Thus, this effect can be considered to be minimal in our study. Second, although all study participants were recruited from one antenatal clinic in an obstetrics and gynecology specialty hospital located in an urban area, high participation rates could have minimized this limitation. In agreement with the findings of previous studies (Jaakkola et al., 2001; Jaddoe et al., 2008; Tsui et al., 2008), our results showed that pregnant women with lower education level and income were more exposed to ETS. Third, due to the low percentage of preterm births, we considered the LBW criteria to include a birth weight of $3,000 \mathrm{~g}$, in addition to $2,500 \mathrm{~g}$.

Regardless of these limitations, our study is the first to show an ETS exposure-related increase in the risk of having an SGA baby. None of the previous prospective studies using biomarkers reported a relationship between ETS exposure and risk of SGA. Furthermore, this is the first study examining ETS effects on perinatal outcomes using hair nicotine concentrations in Asian populations.

The public health consequences of maternal ETS exposure are likely to be extensive. Thus, comprehensive tobacco control policies are needed, especially in Korea.

\section{Acknowledgements}

This study was supported by Wonkwang University in 2013.

\section{References}

Andres RL, Day MC (2000). Perinatal complications associated with maternal tobacco use. Semin Neonatol, 5, 231-41.

Dollberg S, Fainaru O, Mimouni FB, et al (2000). Effect of passive smoking in pregnancy on neonatal nucleated red blood cells. Pediatrics, 106, 34 .

Hughes SC, Corcos IA, Hofstetter CR, et al (2008a). Children's exposure to secondhand smoke at home in Seoul, Korea. Asian Pac J Cancer Prev, 9, 491-5.

Hughes SC, Corcos IA, Hofstetter CR, et al (2008b). Secondhand smoke exposure among nonsmoking adults in Seoul, Korea. Asian Pac J Cancer Prev, 9, 247-52.

Hwang SH, Hwang JH, Moon JS, et al (2012). Environmental tobacco smoke and children's health. Korean J Pediatr, $\mathbf{5 5}, 35-41$.

Jaakkola JJ, Jaakkola N, Zahlsen K (2001). Fetal growth and length of gestation in relation to prenatal exposure to environmental tobacco smoke assessed by hair nicotine concentration. Environ Health Perspect, 109, 557-61.

Jacqz-Aigrain E, Zhang D, Maillard G, et al (2002). Maternal smoking during pregnancy and nicotine and cotinine concentrations in maternal and neonatal hair. BJOG, $\mathbf{1 0 9}$, 909-11.

Jaddoe VW, Troe EJ, Hofman A, et al (2008). Active and passive maternal smoking during pregnancy and the risks of low birthweight and preterm birth: the Generation R Study. Paediatr Perinat Epidemiol, 22, 162-71.

Kang HG, Kwon KH, Lee IW, et al (2013). Biochemicallyverified smoking rate trends and factors associated with inaccurate self-reporting of smoking habits in Korean women. Asian Pac J Cancer Prev, 14, 6807-12.

Kim H, Oh JK, Lim MK, et al (2013). The national "smoking cessation clinics" program in the republic of Korea: socioeconomic status and age matter. Asian Pac J Cancer Prev, 14, 6919-24.

Kim K, Yoo T, Kim Y, et al (2014). Association between cigarette smoking history and mortality in 36,446 health examinees in Korea. Asian Pac J Cancer Prev, 15, 5685-9.

Lee DR, Kim HS, Lee J (2014). The characteristics of false respondents on a self-reported smoking survey of Korean women: Korean national health and nutrition examination survey, 2008. Korean J Fam Med, 35, 28-34.

Lee J (2007). Birth weight for gestational age patterns by sex, plurality, and parity in Korean population. Korean J Pediatr, 50, 732-9.

Leonardi-Bee J, Smyth A, Britton J, et al (2008). Environmental tobacco smoke and fetal health: systematic review and metaanalysis. Arch Dis Child Fetal Neonatal Ed, 93, 351-61.

Lindbohm ML, Sallmen M, Taskinen H (2002). Effects of exposure to environmental tobacco smoke on reproductive health. Scand J Work Environ Health, 28, 84-96.

Misra DP, Nguyen RHN (1999). Environmental tobacco smoke and low birth weight: a hazard in the workplace? Environ Health Perspect, 107, 897-904.

Nafstad P, Fugelseth D, Qvigstad E, et al (1998). Nicotine concentration in the hair of nonsmoking mothers and size of offspring. Am J Public Health, 88, 120-4.

Park EY, Lim MK, Yang W, et al (2013). Policy effects of secondhand smoke exposure in public places in the republic of Korea: evidence from PM2.5 levels and air nicotine concentrations. Asian Pac J Cancer Prev, 14, 7725-30.

Rebagliato M, Florey Cdu V, Bolumar F (1995). Exposure to environmental tobacco smoke in nonsmoking pregnant women in relation to birth weight. Am J Epidemiol, 142, 531-7.

Ryu HJ, Seong MW, Nam MH, et al (2006). Simultaneous and 


\section{Jungun Lee et al}

sensitive measurement of nicotine and cotinine in small amounts of human hair using liquid chromatography/tandem mass spectrometry. Rapid Commun Mass Spectrom, 20, 2781-2.

Salmasi G, Grady R, Jones J, et al (2010). Environmental tobacco smoke exposure and perinatal outcomes: a systematic review and meta-analyses. Acta Obstet Gynecol Scand, 89, 423-41.

Seong MW, Nam MH, Ryu HJ, et al (2007). The comparison of two smoking biomarkers in various biological samples. Clin Chim Acta, 383, 180-1.

Surgeon General's Report (2004). Chapter 5. Reproductive effects. in 'the health consequences of smoking: a report of the surgeon general', eds centers for disease control and prevention (US), Atlanta (GA),

Surgeon General's Report (2006). Chapter 5. Reproductive and developmental effects from exposure to secondhand smokel. in 'the health consequences of involuntary exposure to tobacco smoke: a report of the surgeon general', eds centers for disease control and prevention (US), Atlanta (GA),

Tsui HC, Wu HD, Lin CJ, et al (2008). Prenatal smoking exposure and neonatal DNA damage in relation to birth outcomes. Pediatr Res, 64, 131-4.

Wen CP, Levy DT, Cheng TY, et al (2005). Smoking behaviour in Taiwan, 2001. Tob Control, 14, 51-5.

Yoo SH, Paek YJ, Kim SS, et al (2010). Hair nicotine levels in non-smoking pregnant women whose spouses smoke outside of the home. Tobacco Control, 19, 318-24. 